\title{
ESTABILIDADE DA RESISTÊNCIA DE GENÓTIPOS DE CAUPI A Callosobruchus maculatus (Fabr.) EM GERAÇÕES SUCESSIVAS
}

\author{
Marcileyne Pessôa Leite de Lima ${ }^{1}$; José Vargas de Oliveira ${ }^{1,2 *}$; Reginaldo Barros ${ }^{1}$; Jorge Braz \\ Torres ${ }^{1}$; Manoel Eneas de Carvalho Gonçalves ${ }^{1}$ \\ ${ }^{1}$ Depto. de Agronomia - Fitossanidade/UFRPE, Av. Dom Manoel de Medeiros, s/n, Dois Irmãos - CEP: 52171-900 - \\ Recife, $P E$. \\ ${ }^{2}$ Bolsista CNPq. \\ *Autor correspondente <vargasoliveira@uol.com.br>
}

\begin{abstract}
RESUMO: Callosobruchus maculatus (Fabr.) (Coleoptera: Bruchidae) é a principal praga do caupi (Vigna unguiculata L.) armazenado em condições tropicais e subtropicais. Avaliaram-se a estabilidade da resistência e a capacidade de adaptação de $C$. maculatus a genótipos de caupi, durante seis gerações. Utilizou-se o teste sem chance de escolha, em delineamento experimental inteiramente casualizado, em esquema fatorial, com dez tratamentos, seis gerações do inseto e cinco repetições. Cada repetição constou de 30 grãos infestados com dois casais da praga. O número de ovos/fêmea diferiu entre os genótipos de caupi apenas na sexta geração, e entre as gerações em BR14-Mulato, Bico de Pato, TE90-180-3E e TE87-98-8G. A viabilidade de ovos diferiu entre as gerações em BR17-Gurguéia, BR14-Mulato, IT89KD-260 e IT89KD245 , e entre os genótipos nas terceira, quinta e sexta gerações. Os genótipos diferiram entre e dentro das gerações, em relação à duração e a viabilidade da fase imatura. Observou-se redução na emergência, especialmente em IT89KD-245, IT89KD-260, CNC 0434, Bico de Pato, TE90-180-10F e BR14-Mulato, provavelmente devido a substâncias químicas presentes nos grãos que afetaram a sobrevivência dos insetos, ao longo das gerações. Os genótipos IT89KD-245 e IT89KD-260 comportaram-se como moderadamente resistentes. Não se observou adaptação de C. maculatus aos genótipos, mantendo-se a resistência estável através das gerações.
\end{abstract}

Palavras-chave: Vigna unguiculata, insecta, resistência de plantas, antibiose

\section{STABILITY OF THE RESISTANCE OF COWPEA GENOTYPES TO Callosobruchus maculatus (Fabr.) IN SUCCESSIVE GENERATIONS}

\begin{abstract}
Callosobruchus maculatus (Fabr.) (Coleoptera: Bruchidae) is the most important pest of stored cowpea in tropical and subtropical conditions. This paper evaluates the stability of the resistance and the capacity of adaptation of $C$. maculatus to cowpea genotypes for six generations. A test without choice chance was used, in a factorial, completely randomized design, with ten treatments $(n=5)$ in six generations of the insect. Each replication was represented by 30 grains infested by two pairs of the pest. The number of eggs/ female only differed between the cowpea genotypes in the sixth generation, and between generations in BR14-Mulato, Bico de Pato, TE90-180-3E and TE87-98-8G. Viability of eggs differed between generations for BR17-Gurguéia, BR14-Mulato, IT89KD-260 and IT89KD-245, and between the genotypes in the third, fifth and sixth generations. The genotypes differed between and within generations in regard to the duration and viability of the immature phase. A reduction in the emergency, especially on IT89KD-245, IT89KD-260, CNC 0434, Bico de Pato, TE90-180-10F and BR14-Mulato, probably due to chemical substances present in the grains that affected the survival of the insects, was observed throughout generations. Genotypes IT89KD-245 and IT89KD-260 standed as moderately resistant. The adaptation of C. maculatus to the genotypes was not observed, which remained resistance throughout generations.
\end{abstract}

Key words: Vigna unguiculata, insecta, plant resistance, antibiosis

\section{INTRODUÇÃO}

O caruncho Callosobruchus maculatus (Fabr.) (Coleoptera: Bruchidae) é uma das principais pragas de várias espécies de Vigna, em especial de $V$. unguiculata (caupi), que é uma importante fonte de proteína em regiões tropicais e subtropicais. A infestação deste bruquídeo começa no campo e continua no armazém, podendo danificar totalmente os grãos após cinco meses (Singh, 1978; Messina \& Renwick, 1985; Mbata, 1993).
Nos países produtores de caupi, o uso de cultivares resistentes constitui uma alternativa promissora, com o objetivo de reduzir os danos e as perdas no armazenamento. Deste modo, várias pesquisas têm procurado selecionar fontes de resistência a C. maculatus (International Institute of Tropical Agriculture (IITA), 1990; Mbata, 1993; Seck, 1993; Chaves \& Vendramim, 1995), embora poucas fontes com alta resistência tenham sido identificadas, o que tem dificultado o desenvolvimento de cultivares resistentes (Singh, 1978; IITA,1981; Singh et al., 1985; IITA, 1990). 
A resistência de cultivares de caupi pode ser suplantada por biótipos de C. maculatus, capazes de alterar a relação planta - inseto. Ainda que a utilização da planta pela praga se mantenha em níveis baixos por várias gerações, a intensidade da pressão seletiva produziria uma população de insetos adaptados, tal como foi constatado por Redden et al. (1983), Dick \& Credland (1986a) e Shade et al. (1996). A mudança da resposta de C. maculatus ao cultivar resistente TVu 2027 foi demonstrada por Dick \& Credland (1986a) em três gerações de biótipos do Yemen e da Nigéria, onde o desenvolvimento dos insetos ocorreu rapidamente, levando a uma evolução da população capaz de sobreviver neste cultivar. Assim, a manutenção da resistência durante períodos prolongados, será condicionada ao surgimento de outros biótipos (Beck \& Schoonhover, 1984). Neste trabalho estudou-se o efeito de genótipos de caupi com diferentes graus de resistência na biologia de $C$. maculatus, durante seis gerações, visando avaliar a estabilidade da resistência e a capacidade de adaptação do inseto.

\section{MATERIAL E MÉTODOS}

A pesquisa foi desenvolvida no Laboratório de Entomologia Agrícola da Área de Fitossanidade da Universidade Federal Rural de Pernambuco, à temperatura de $27,1 \pm 0,9^{\circ} \mathrm{C}$, umidade relativa de $66,6 \pm$ $4 \%$, registradas diariamente em termohigrógrafo e fotofase de $12 \mathrm{~h}$.

Foram utilizados dez genótipos (cultivares e linhagens) de caupi procedentes da EMBRAPA/CPAMN, Teresina-PI, e multiplicados na Estação Experimental Luiz Jorge da Gama Wanderley - Empresa Pernambucana de Pesquisa Agropecuária (IPA), Vitória de Santo Antão-PE. Após a colheita e beneficiamento, os grãos foram acondicionados em sacos plásticos em congelador a temperatura de $-5^{\circ} \mathrm{C}$ para eliminação de eventuais infestações de $C$. maculatus no campo. Antes da instalação dos experimentos, os grãos foram retirados do congelador, colocados em recipientes plásticos cobertos com tecido fino por, no mínimo, seis dias para entrarem em equilíbrio higroscópico.

A escolha dos genótipos foi realizada com base nos resultados obtidos por Lima et al. (no prelo) ${ }^{1}$, durante uma geração de C. maculatus. Selecionaram-se cinco genótipos MR = moderadamente resistentes (IT89KD245, TE90-180-10F, CNCx 409-12F, IT89KD-260 e BR17Gurguéia), três $S=$ suscetíveis (CNC 0434, BR14-Mulato e TE87-98-8G) e dois AS = altamente suscetíveis (TE90180-3E e Bico de Pato), de acordo com o índice de resistência de Kornegay et al.(1993).

Os insetos criados em grãos de cada genótipo, constituindo a geração parental, foram retirados no quarto dia de emergência e usados na infestação da primeira geração no mesmo genótipo. Trinta grãos íntegros de cada genótipo de caupi, infestados com dois casais de C. maculatus com 0-24 horas de idade durante seis dias para oviposição (Santos, 1976), foram acondicionados em recipientes plásticos de $6 \mathrm{~cm}$ de altura e $6 \mathrm{~cm}$ de diâmetro, com tampas perfuradas e recobertas com tecido fino para favorecer a aeração. Após este período, os insetos foram descartados e os grãos mantidos no laboratório para as devidas observações. A partir do vigésimo quinto dia após a infestação (DAl), os grãos foram examinados diariamente para observação da emergência dos adultos, os quais foram contados e removidos. A inspeção continuou por um período de 23 dias, tempo suficiente para que todos os insetos emergissem. O mesmo procedimento foi realizado nas seis gerações.

$\mathrm{Na}$ avaliação do efeito dos genótipos foram utilizados os parâmetros número de ovos/fêmea (fecundidade), viabilidade de ovos (fertilidade), duração da fase imatura (período de ovo a adulto), viabilidade da fase imatura (porcentagem de adultos emergidos) e índice de resistência. A viabilidade de ovos foi calculada a partir do número de ovos viáveis em relação ao total de ovos, sendo os mesmos distinguidos pela aparência opaca, devido aos resíduos liberados pela larva durante a penetração no grão. Para cálculo da duração da fase imatura, utilizou-se: [ $\Sigma$ (número diário de insetos emergidos $x$ número de DAI)/total de insetos emergidos]. A viabilidade da fase imatura foi obtida mediante o total de insetos emergidos em relação ao número de ovos viáveis. O índice de resistência foi calculado pela seguinte fórmula: In \{[(número de insetos emergidos/ número de ovos viáveis) +1 /duração da fase imatura] $x$ $100\}$ (Kornegay et al., 1993).

O delineamento experimental adotado foi inteiramente casualizado, em esquema fatorial, com dez tratamentos, seis gerações da praga e cinco repetições. Os dados referentes ao número de ovos/fêmea, viabilidade de ovos, duração e viabilidade da fase imatura foram submetidos ao teste de Lilliefors para normalidade de variância, à análise de variância (ANOVA) e ao teste de Scott-Knott $(P \leq 0,05)$ para separação de médias (Scott \& Knott, 1974). Os genótipos dentro de uma mesma geração, quando avaliados pelo índice de resistência (Kornegay et al., 1993), foram separados pelo intervalo de confiança (IC) $(P=0,05)$ (Barros \& Vendramim, 1999) e agrupados em três graus de resistência: MR (Mc/l < Mc/lt - IC), S (Mc/lt $-\mathrm{IC} \leq \mathrm{Mc} /$ $\mathrm{I} \leq \mathrm{Mc} / \mathrm{lt}+\mathrm{IC})$ e AS $(\mathrm{Mc} / \mathrm{l}>\mathrm{Mc} / \mathrm{lt}+\mathrm{IC})$; sendo $\mathrm{Mc} / \mathrm{l} \mathrm{a}$ média do genótipo, e Mc/lt a média dos genótipos testados. Determinaram-se, também, correlações simples entre todos os parâmetros em cada geração.

\section{RESULTADOS E DISCUSSÃO}

O número de ovos por fêmea de $C$. maculatus somente diferiu entre os genótipos de caupi na sexta geração, e entre as gerações em BR14-Mulato, Bico de Pato, TE90-180-3E e TE87-98-8G. Nestas, ao longo das 
gerações, observou-se uma redução no número de ovos. Os fatores químicos presentes nos grãos que afetaram a fecundidade da praga não foram estudados neste trabalho. Na média das seis gerações, o menor número de ovos foi obtido em IT89KD-260, IT89KD-245 e BR17-Gurguéia (Tabela 1).

A viabilidade média de ovos apresentou pequena variação entre os genótipos. Entre as gerações, só houve diferença para BR17-Gurguéia, BR14-Mulato, IT89KD260 e IT89KD-245, e entre os genótipos nas terceira, quinta e sexta gerações. A menor viabilidade de ovos foi verificada em BR14-Mulato por cinco gerações, no entanto desconhece-se as características morfológicas do grão que dificultaram a penetração, ou químicas que proporcionaram alta mortalidade das larvas recémeclodidas (Tabela 2).

Os genótipos diferiram entre e dentro das gerações, quanto à duração da fase imatura. A maior duração média foi observada em IT89KD-245 (30,5 dias) e a menor em TE90-180-3E (29,4 dias), correspondendo a apenas 1,1 dia de diferença (Tabela 3 ). A amplitude de um dia também foi observada por Santos (1976) e Pessoa et al. (1993), em relação a outros cultivares de caupi para o período de ovo a adulto. Por outro lado, Baker et al. (1989) observaram uma amplitude superior a 30 dias, em linhagens suscetíveis e resistentes provenientes do IITA. Observou-se em IT89KD-245, por cinco gerações, a maior duração desta fase, onde na terceira geração a amplitude chegou a 1,6 dias em relação a TE90-180-3E (Tabela 3), demonstrando que a primeira foi menos favorável ao desenvolvimento de $C$. maculatus, por reduzir o número de gerações anuais. IT89KD-245 e CNC 0434 foram os genótipos que mais alongaram a fase imatura da praga dentro de cada uma das seis gerações, embora não se conheça as substâncias químicas responsáveis presentes nos grãos.

A viabilidade da fase imatura variou entre os genótipos dentro e entre as gerações, constatando-se redução na emergência, ao longo das gerações especialmente em IT89KD-245, IT89KD-260, CNC 0434, Bico de Pato, TE90-180-10F e BR14-Mulato. Na média das gerações, a variação foi de 68,9\% em IT89KD-245

Tabela 1 - Número de ovos por fêmea (Média \pm IC) de $C$. maculatus, em genótipos de caupi, por seis gerações sucessivas. Temperatura: $27,1 \pm 0,9^{\circ} \mathrm{C}$; UR: $66,6 \pm 4 \%$; Fotofase: $12 \mathrm{~h}$.

\begin{tabular}{|c|c|c|c|c|c|c|c|}
\hline \multirow{2}{*}{ Genótipo } & \multicolumn{7}{|c|}{ Geração } \\
\hline & $1^{\mathrm{a}}$ & $2^{a}$ & $3^{a}$ & $4^{a}$ & $5^{a}$ & $6^{a}$ & Média \\
\hline TE87-98-8G & $90,9 \pm 5,31 \mathrm{aA}$ & $86,4 \pm 6,00 \mathrm{aA}$ & $86,0 \pm 4,15 \mathrm{aA}$ & $83,2 \pm 6,36$ aA & $83,9 \pm 3,61 \mathrm{aA}$ & $70,9 \pm 13,85 b B$ & $83,6 \pm 5,41 a$ \\
\hline Bico de Pato & $84,6 \pm 8,62 \mathrm{aA}$ & $85,4 \pm 1,97 \mathrm{aA}$ & $88,1 \pm 7,05 \mathrm{aA}$ & $84,7 \pm 5,05 \mathrm{aA}$ & $81,8 \pm 3,32 \mathrm{aA}$ & $68,6 \pm 11,13 b B$ & $82,2 \pm 5,57 \mathrm{a}$ \\
\hline CNCx 409-12F & $86,5 \pm 7,03 \mathrm{aA}$ & $73,2 \pm 12,39 \mathrm{aA}$ & $80,5 \pm 4,29 a A$ & $79,8 \pm 5,56 \mathrm{aA}$ & $80,0 \pm 3,88 \mathrm{aA}$ & $83,6 \pm \quad 4,76 \mathrm{aA}$ & $80,6 \pm 3,57 a$ \\
\hline CNC 0434 & $80,8 \pm 7,63 \mathrm{aA}$ & $80,8 \pm 4,41 \mathrm{aA}$ & $82,1 \pm 7,39 \mathrm{aA}$ & $82,5 \pm 4,43 \mathrm{aA}$ & $78,2 \pm 3,50 \mathrm{aA}$ & $77,3 \pm 6,43 a A$ & $80,3 \pm 1,68 a$ \\
\hline TE $90-180-3 E$ & $85,5 \pm 5,55 \mathrm{aA}$ & $83,5 \pm 5,81 \mathrm{aA}$ & $82,9 \pm 4,24 \mathrm{aA}$ & $80,9 \pm 3,24 \mathrm{aA}$ & $75,2 \pm 5,03 \mathrm{aB}$ & $71,7 \pm 14,29$ bB & $80,0 \pm 4,29 a$ \\
\hline TE90-180-10F & $86,3 \pm 4,75 a A$ & $74,9 \pm 5,81 \mathrm{aA}$ & $82,4 \pm 2,98 \mathrm{aA}$ & $80,5 \pm 4,44 \mathrm{aA}$ & $78,4 \pm 4,57 \mathrm{aA}$ & $74,6 \pm 10,98 \mathrm{aA}$ & $79,5 \pm 3,61 a$ \\
\hline BR14-Mulato & $86,0 \pm 7,61 \mathrm{aA}$ & $83,9 \pm 9,21 \mathrm{aA}$ & $82,7 \pm 5,39 \mathrm{aA}$ & $70,4 \pm 6,07 \mathrm{aB}$ & $78,8 \pm 4,32 \mathrm{aB}$ & $76,7 \pm 2,69 a \mathrm{aB}$ & $79,8 \pm 4,56 a$ \\
\hline IT89KD-260 & $76,2 \pm 16,71 \mathrm{aA}$ & $74,4 \pm 6,64 \mathrm{aA}$ & $77,7 \pm 5,34 \mathrm{aA}$ & $78,7 \pm 8,66 \mathrm{aA}$ & $72,2 \pm 3,65 \mathrm{aA}$ & $80,2 \pm 5,68 \mathrm{aA}$ & $76,6 \pm 2,34 b$ \\
\hline IT89KD-245 & $81,2 \pm 4,15$ aA & $75,7 \pm 2,95 \mathrm{aA}$ & $78,1 \pm 4,98 \mathrm{aA}$ & $72,3 \pm 3,05 a A$ & $74,6 \pm 3,79 a A$ & $69,8 \pm 10,29 b A$ & $75,3 \pm 3,25 b$ \\
\hline BR17-Gurguéia & $83,1 \pm 12,46 \mathrm{aA}$ & $77,3 \pm 11,92 \mathrm{aA}$ & $74,8 \pm 6,46 a A$ & $76,8 \pm 2,98 \mathrm{aA}$ & $72,6 \pm 5,18 \mathrm{aA}$ & $66,8 \pm 8,54 \mathrm{aA}$ & $75,2 \pm 4,34 b$ \\
\hline
\end{tabular}

Tabela 2 - Viabilidade de ovos (Média $\pm I C$ ) de C. maculatus, em genótipos de caupi, por seis gerações sucessivas. Temperatura: $27,1 \pm 0,9^{\circ} \mathrm{C}$; UR: 66,6 \pm 4\%; Fotofase: $12 \mathrm{~h}$.

\begin{tabular}{|c|c|c|c|c|c|c|c|}
\hline \multirow{2}{*}{ Genótipo } & \multicolumn{7}{|c|}{ Geração } \\
\hline & $1^{\mathrm{a}}$ & $2^{\mathrm{a}}$ & $3^{a}$ & $4^{a}$ & $5^{a}$ & $6^{a}$ & Média \\
\hline & \multicolumn{7}{|c|}{ 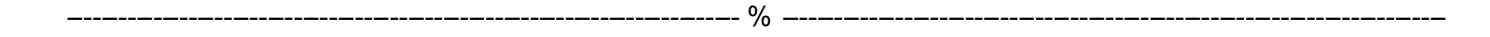 } \\
\hline $\mathrm{CNCx} 409-12 \mathrm{~F}$ & $94,2 \pm 3,22 \mathrm{aA}$ & $97,0 \pm 1,10 \mathrm{aA}$ & $94,8 \pm 1,37$ aA & $95,7 \pm 4,52 \mathrm{aA}$ & $92,3 \pm 1,97$ aA & $94,8 \pm 1,54 \mathrm{aA}$ & $94,8 \pm 1,25 a$ \\
\hline Bico de Pato & $89,8 \pm 7,34 \mathrm{aA}$ & $92,5 \pm 6,89 \mathrm{aA}$ & $96,1 \pm 1,78 \mathrm{aA}$ & $97,4 \pm 1,21 \mathrm{aA}$ & $95,1 \pm 2,52 \mathrm{aA}$ & $95,0 \pm 1,66 \mathrm{aA}$ & $94,3 \pm 2,19 a$ \\
\hline CNC 0434 & $92,0 \pm 2,89 \mathrm{aA}$ & $95,4 \pm 1,31 \mathrm{aA}$ & $93,8 \pm 2,69 \mathrm{aA}$ & $96,5 \pm 1,06 \mathrm{aA}$ & $91,2 \pm 1,66$ aA & $92,2 \pm 2,09 \mathrm{aA}$ & $93,5 \pm 1,38 a$ \\
\hline IT89KD-245 & $92,2 \pm 4,61 \mathrm{aA}$ & $92,4 \pm 5,47 \mathrm{aA}$ & $94,6 \pm 4,39 \mathrm{aA}$ & $97,5 \pm 0,85 \mathrm{aA}$ & $93,8 \pm 1,78$ aA & $84,4 \pm 9,34 \mathrm{bB}$ & $92,5 \pm 3,52 \mathrm{a}$ \\
\hline BR17-Gurguéia & $93,9 \pm 3,78$ aA & $93,4 \pm 3,83 \mathrm{aA}$ & $84,0 \pm 9,29 \mathrm{bB}$ & $95,3 \pm 2,38$ aA & $92,7 \pm 2,94 \mathrm{aA}$ & $94,2 \pm 1,83 \mathrm{aA}$ & $92,3 \pm 3,31 a$ \\
\hline TE $90-180-3 E$ & $95,2 \pm 2,65 \mathrm{aA}$ & $93,1 \pm 4,48$ aA & $92,0 \pm 2,99 \mathrm{aA}$ & $92,4 \pm 4,04 \mathrm{aA}$ & $86,7 \pm 6,44 \mathrm{bA}$ & $94,0 \pm 3,30 \mathrm{aA}$ & $92,2 \pm 2,36 a$ \\
\hline TE90-180-1 OF & $90,4 \pm 5,14$ aA & $92,9 \pm 3,85 a A$ & $93,7 \pm 1,97 \mathrm{aA}$ & $93,6 \pm 2,40 \mathrm{aA}$ & $88,7 \pm 5,19 \mathrm{bA}$ & $91,6 \pm 3,27 \mathrm{aA}$ & $91,8 \pm 1,59 a$ \\
\hline TE87-98-8G & $93,1 \pm 3,31 \mathrm{aA}$ & $94,0 \pm 3,87$ aA & $92,7 \pm 1,25$ aA & $92,4 \pm 3,84 \mathrm{aA}$ & $90,6 \pm 2,49$ aA & $86,9 \pm 8,61 \mathrm{bA}$ & $91,6 \pm 2,05 a$ \\
\hline IT89KD-260 & $91,2 \pm 4,51 \mathrm{aA}$ & $93,7 \pm 3,41 \mathrm{aA}$ & $90,0 \pm 5,81 \mathrm{bA}$ & $93,6 \pm 2,07 \mathrm{aA}$ & $92,0 \pm 2,51 \mathrm{aA}$ & $84,7 \pm 5,12$ bB & $90,9 \pm 2,67 a$ \\
\hline BR14-Mulato & $89,4 \pm 6,12 \mathrm{aA}$ & $89,7 \pm 4,05 \mathrm{aA}$ & $87,9 \pm 5,60 \mathrm{bA}$ & $89,7 \pm 5,08 \mathrm{aA}$ & $83,1 \pm 4,79 \mathrm{bB}$ & $75,8 \pm 7,24 \mathrm{cC}$ & $85,9 \pm 4,45 b$ \\
\hline
\end{tabular}


a $84,8 \%$ em CNCx 409-12F, correspondendo a reduções de $31,1 \%$ e $15,2 \%$ na progênie de $C$. maculatus, respectivamente (Tabela 4), devido a presença de substâncias químicas com ação antibiótica presentes nos grãos que não foram quantificadas.

Com base no índice de resistência médio (Kornegay et al., 1993), os genótipos IT89KD-245 e IT89KD-260 destacaram-se como MR. Todavia, TE90180-10F, BR17-Gurguéia e CNCx 409-12F, classificados como MR em uma seleção anterior, não se mantiveram nesta classificação ao longo das seis gerações, comportando-se como S, S e AS, respectivamente. Esta mudança na classificação não pode ser considerada como uma adaptação de $C$. maculatus, tendo em vista que os genótipos não apresentaram alto nível de resistência. Já Bico de Pato, considerado como AS, manteve-se neste grau de resistência apenas nas duas primeiras gerações, e como MR nas três subsequentes, caracterizando talvez um efeito cumulativo da resistência. Os genótipos CNCx 409-12F e IT89KD-245 foram os únicos que se mantiveram no mesmo grau de resistência nas seis gerações (Tabela 5).
O genótipo IT89KD-245 destacou-se pela sua menor viabilidade e a maior duração média da fase imatura, indicando ser desfavorável ao desenvolvimento de C. maculatus e, portanto, portador de resistência por antibiose. O inverso foi observado em CNCx 409-12F, com maiores valores médios de viabilidade de ovos e da fase imatura e uma das menores durações desta fase, demonstrando ser mais suscetível à praga.

Os coeficientes de correlação entre a viabilidade da fase imatura e o índice de resistência foram superiores a $0,95 \mathrm{em}$ todas as gerações, evidenciando desfavorecimento na relação genótipo - C. maculatus e, portanto, considerados os parâmetros mais consistentes neste estudo. As correlações por serem positivas indicam que os genótipos que reduziram a sobrevivência dos insetos causaram, ao mesmo tempo, a redução deste índice. Entre a duração da fase imatura e o índice de resistência só houve correlação na primeira e sexta gerações, e por serem negativas implica que o pior desenvolvimento da praga (maior duração da fase imatura) foi observado nos genótipos com menor índice de resistência (Tabela 6).

Tabela 3 - Duração da fase imatura (Média $\pm I C$ ) de C. maculatus, em genótipos de caupi, por seis gerações sucessivas. Temperatura: $27,1 \pm 0,9^{\circ} \mathrm{C}$; UR: $66,6 \pm 4 \%$; Fotofase: $12 \mathrm{~h}$.

\begin{tabular}{|c|c|c|c|c|c|c|c|}
\hline \multirow{2}{*}{ Ge nótipo } & \multicolumn{7}{|c|}{ Geração } \\
\hline & $1^{\mathrm{a}}$ & $2^{a}$ & $3^{a}$ & $4^{a}$ & $5^{\mathrm{a}}$ & $6^{\mathrm{a}}$ & Média \\
\hline & \multicolumn{7}{|c|}{ 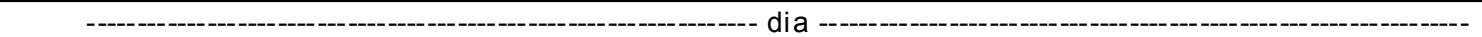 } \\
\hline IT89KD-245 & $30,2 \pm 0,57 \mathrm{aC}$ & $31,8 \pm 0,31 \mathrm{aA}$ & $31,2 \pm 0,69 \mathrm{aB}$ & $31,0 \pm 0,29 \mathrm{aB}$ & $29,7 \pm 0,31 \mathrm{aD}$ & $29,2 \pm 0,39 \mathrm{aD}$ & $30,5 \pm 0,79 a$ \\
\hline CNC 0434 & $29,3 \pm 0,13 b D$ & $31,6 \pm 0,41 \mathrm{aA}$ & $30,4 \pm 0,44 \mathrm{bC}$ & $30,7 \pm 0,37 \mathrm{aB}$ & $30,0 \pm 0,30 \mathrm{aC}$ & $28,7 \pm 0,25 \mathrm{aE}$ & $30,1 \pm 0,82$ \\
\hline Bico de Pato & $29,3 \pm 0,34 \mathrm{bC}$ & $31,3 \pm 0,32 \mathrm{bA}$ & $30,2 \pm 0,16 b B$ & $30,5 \pm 0,36 \mathrm{aB}$ & $29,6 \pm 0,15 \mathrm{aC}$ & $29,1 \pm 0,39 \mathrm{aC}$ & $30,0 \pm 0,66 b$ \\
\hline BR14-Mulato & $29,3 \pm 0,40 \mathrm{bC}$ & $31,1 \pm 0,45 b A$ & $30,1 \pm 0,31 \mathrm{bB}$ & $30,4 \pm 0,36 \mathrm{aB}$ & $29,7 \pm 0,23 \mathrm{aC}$ & $28,7 \pm 0,13 \mathrm{aD}$ & $29,9 \pm 0,68 c$ \\
\hline CNCx 409-12F & $29,0 \pm 0,25 \mathrm{cC}$ & $31,0 \pm 0,35 b A$ & $30,5 \pm 0,32 b B$ & $30,4 \pm 0,23 \mathrm{aB}$ & $29,3 \pm 0,23 b C$ & $28,3 \pm 0,25 b B$ & $29,8 \pm 0,83 c$ \\
\hline TE90-180-10F & $29,0 \pm 0,35 \mathrm{cC}$ & $30,9 \pm 0,12 b A$ & $30,2 \pm 0,12 b B$ & $30,4 \pm 0,13 \mathrm{aB}$ & $29,4 \pm 0,19 b C$ & $28,3 \pm 0,27 b D$ & $29,7 \pm 0,78 c$ \\
\hline BR17-Gurguéia & $29,4 \pm 0,39 b C$ & $30,9 \pm 0,33 \mathrm{bA}$ & $30,2 \pm 0,27 b B$ & $30,1 \pm 0,16 \mathrm{bB}$ & $29,4 \pm 0,20 \mathrm{bC}$ & $28,2 \pm 0,16 b D$ & $29,7 \pm 0,74 \mathrm{c}$ \\
\hline TE87-98-8G & $28,8 \pm 0,25 \mathrm{cD}$ & $30,9 \pm 0,29 b A$ & $30,1 \pm 0,21 b B$ & $30,4 \pm 0,33 \mathrm{aB}$ & $29,3 \pm 0,29 b C$ & $28,8 \pm 1,18 \mathrm{aD}$ & $29,7 \pm 0,70 \mathrm{c}$ \\
\hline IT89KD-260 & $28,9 \pm 0,34 \mathrm{cD}$ & $31,1 \pm 0,38 \mathrm{bA}$ & $29,6 \pm 0,16 \mathrm{cC}$ & $30,2 \pm 0,52 b B$ & $29,2 \pm 0,16 b C$ & $28,8 \pm 0,34 a D$ & $29,6 \pm 0,71 c$ \\
\hline TE $90-180-3 E$ & $28,7 \pm 0,15 \mathrm{cC}$ & $30,7 \pm 0,40 \mathrm{bA}$ & $29,8 \pm 0,41 \mathrm{cB}$ & $29,7 \pm 0,22 \mathrm{bB}$ & $29,1 \pm 0,31 b C$ & $28,3 \pm 0,28 b D$ & $29,4 \pm 0,69 c$ \\
\hline
\end{tabular}

${ }^{1}$ Médias seguidas de mesma letra minúscula, na coluna, e maiúscula, na linha, não diferem pelo teste de Scott-Knott $(P \leq 0,05)$.

Tabela 4 - Viabilidade da fase imatura (Média $\pm \mathrm{IC}$ ) de $C$. maculatus, em genótipos de caupi, por seis gerações sucessivas. Temperatura: $27,1 \pm 0,9^{\circ} \mathrm{C}$; UR: $66,6 \pm 4 \%$; Fotofase: $12 \mathrm{~h}$.

\begin{tabular}{|c|c|c|c|c|c|c|c|}
\hline \multirow{2}{*}{ Ge nótipo } & \multicolumn{7}{|c|}{ Geração } \\
\hline & $1^{\mathrm{a}}$ & $2^{\mathrm{a}}$ & $3^{\mathrm{a}}$ & $4^{\mathrm{a}}$ & $5^{\mathrm{a}}$ & $6^{\mathrm{a}}$ & Média \\
\hline & \multicolumn{7}{|c|}{ - } \\
\hline CNCX 409-12F & $88,1 \pm 3,01$ aA & $81,7 \pm 6,81 \mathrm{aA}$ & $86,0 \pm 7,57$ aA & $84,4 \pm 3,26 \mathrm{bA}$ & $85,6 \pm 2,86$ aA & $83,0 \pm 3,41 \mathrm{aA}$ & $84,8 \pm 1,82 \mathrm{a}$ \\
\hline TE $90-180-3 E$ & $85,4 \pm 7,24 \mathrm{aA}$ & $78,1 \pm 5,80 \mathrm{bA}$ & $82,0 \pm 7,59 \mathrm{aA}$ & $82,9 \pm 8,98$ aA & $83,1 \pm 6,22 \mathrm{aA}$ & $79,7 \pm 7,23 \mathrm{aA}$ & $81,9 \pm 2,09 a$ \\
\hline BR17-Gurguéia & $79,6 \pm 3,59 b A$ & $78,2 \pm 8,07 \mathrm{bA}$ & $80,4 \pm 6,67 \mathrm{aA}$ & $80,8 \pm 6,10 \mathrm{aA}$ & $82,8 \pm 3,00 \mathrm{aA}$ & $76,0 \pm 8,78 a A$ & $79,6 \pm 1,87 a$ \\
\hline TE 87-98-8G & $87,4 \pm 2,56 a A$ & $77,4 \pm 2,51 \mathrm{bA}$ & $80,4 \pm 5,20 \mathrm{aA}$ & $78,9 \pm 4,18$ aA & $80,4 \pm 6,23 \mathrm{aA}$ & $70,9 \pm 12,71 \mathrm{bB}$ & $79,2 \pm 4,27 a$ \\
\hline CNC 0434 & $95,1 \pm 4,61 \mathrm{aA}$ & $72,3 \pm 6,01 \mathrm{bB}$ & $79,4 \pm 3,28 \mathrm{aA}$ & $72,7 \pm 3,43 \mathrm{bB}$ & $75,0 \pm 3,01 \mathrm{bB}$ & $65,3 \pm 3,53 b B$ & $76,6 \pm 8,11 b$ \\
\hline Bico de Pato & $91,2 \pm 1,67 \mathrm{aA}$ & $85,6 \pm 5,42 \mathrm{aA}$ & $73,3 \pm 2,77 \mathrm{bB}$ & $66,7 \pm 5,79 \mathrm{cB}$ & $67,4 \pm 3,76 \mathrm{cB}$ & $68,6 \pm 8,92 \mathrm{bB}$ & $75,5 \pm 8,35 b$ \\
\hline BR14-Mulato & $79,9 \pm 3,53 b A$ & $71,6 \pm 5,68 \mathrm{bB}$ & $73,8 \pm 1,88 \mathrm{bA}$ & $81,8 \pm 5,54 \mathrm{aA}$ & $76,1 \pm 5,97 \mathrm{bA}$ & $63,9 \pm 6,10 \mathrm{bB}$ & $74,5 \pm 5,14 b$ \\
\hline TE90-180-10F & $85,1 \pm 3,21 \mathrm{aA}$ & $76,1 \pm 3,96 \mathrm{bB}$ & $75,5 \pm 5,72 \mathrm{bB}$ & $70,3 \pm 5,52 \mathrm{bC}$ & $73,6 \pm 5,57 \mathrm{bB}$ & $62,5 \pm 6,30 \mathrm{bC}$ & $73,9 \pm 5,94 b$ \\
\hline IT89KD-260 & $85,3 \pm 5,49 a A$ & $73,8 \pm 2,50 \mathrm{bB}$ & $73,6 \pm 5,39 \mathrm{bB}$ & $62,8 \pm 7,12 \mathrm{cC}$ & $76,0 \pm 5,97 \mathrm{bB}$ & $61,8 \pm 10,07 \mathrm{bC}$ & $72,2 \pm 7,04 b$ \\
\hline IT89KD-245 & $79,9 \pm 4,47 \mathrm{bA}$ & $73,8 \pm 4,25 \mathrm{bA}$ & $74,3 \pm 11,14 \mathrm{bA}$ & $68,4 \pm 4,08 \mathrm{aA}$ & $53,1 \pm 8,29 \mathrm{dC}$ & $7,88 \mathrm{bB}$ & $68,9 \pm 7,60 \mathrm{c}$ \\
\hline
\end{tabular}


$\mathrm{Na}$ avaliação da resistência de grãos de caupi a C. maculatus, a viabilidade e a duração da fase imatura são considerados os parâmetros m ais importantes (Redden \& McGuire, 1983). No entanto, neste trabalho, o índice de resistência que também utiliza no seu cálculo estes dois parâmetros, mostrouse mais apropriado. Além disso, uma estimativa numérica simples da performance de $C$. maculatus é conveniente e mais válido do que usar um único parâmetro isoladamente.

A baixa porcentagem de emergência, resultante de uma pequena sobrevivência larval, reduzirá o número de descendentes que contribuiriam para o crescimento populacional de geração a geração (Ofuya \& Credland, 1995). A habilidade de um hospedeiro resistente em retardar o desenvolvimento de pragas, indica que a taxa de multiplicação ou aumento do número de insetos em populações naturais será reduzida devido ao maior tempo médio de cada geração. Este fato é particularmente importante em C. maculatus, segundo Shade et al. (1996), pois como uma espécie multivoltina pode apresentar 10 a 12 gerações anuais em regiões tropicais e subtropicais.

Apesar de não ter havido alta mortalidade larval nos genótipos classificados como resistentes, aliada ao atraso no desenvolvimento, pode levar à considerável redução nas perdas de grãos durante o armazenamento, resultado também obtido por Messina \& Renwick (1985), em linhagens resistentes de caupi.

A criação contínua de $C$. maculatus em cultivares de caupi resistentes pode resultar em aumento progressivo na freqüência de genes de resistência nos insetos e, consequentemente, melhorar o seu desempenho frente os cultivares (Dick \& Credland, 1986b). Não obstante, a variação geográfica entre populações desta praga tem implicações importantes, a curto prazo, para o sucesso de cultivares de caupi resistentes produzidas para distribuição mundial, já que

Tabela 5 - Índice de resistência de C. maculatus, em genótipos de caupi, por seis gerações sucessivas. Temperatura: 27,1 $\pm 0,9^{\circ} \mathrm{C}$; UR: $66,6 \pm 4 \%$; Fotofase: $12 \mathrm{~h}$.

\begin{tabular}{|c|c|c|c|c|c|c|c|}
\hline \multirow{2}{*}{ Genótipo } & \multicolumn{7}{|c|}{ Geração o, } \\
\hline & $1^{\mathrm{a}}$ & $2^{a}$ & $3^{a}$ & $4^{a}$ & $5^{a}$ & $6^{a}$ & Média \\
\hline CNCx 409-12F & $2,18 \mathrm{AS}$ & 1,98 AS & $2,03 \mathrm{AS}$ & $2,01 \mathrm{AS}$ & $2,11 \mathrm{AS}$ & 2,14 AS & $2,07 \mathrm{AS}$ \\
\hline TE $90-180-3 E$ & $2,15 \mathrm{~S}$ & $1,88 \mathrm{~S}$ & $2,01 \mathrm{AS}$ & 2,02 AS & 2,09 AS & 2,07 AS & 2,04 AS \\
\hline BR17-Gurguéia & 1,98 MR & $1,85 \mathrm{~S}$ & $1,94 \mathrm{~S}$ & $1,97 \mathrm{AS}$ & 2,05 AS & $2,00 \mathrm{AS}$ & $1,97 \mathrm{~S}$ \\
\hline TE 87-98-8G & $2,18 \mathrm{AS}$ & $1,86 \mathrm{~S}$ & 1,96 AS & $1,92 \mathrm{~S}$ & $2,02 \mathrm{~S}$ & $1,84 \mathrm{~S}$ & $1,96 \mathrm{~S}$ \\
\hline Bico de Pato & $2,21 \mathrm{AS}$ & $1,98 \mathrm{AS}$ & 1,82 MR & 1,67 MR & 1,74 MR & $1,82 \mathrm{~S}$ & $1,87 \mathrm{~S}$ \\
\hline BR14-Mulato & 2,01 MR & 1,74 MR & 1,84 MR & $1,96 \mathrm{AS}$ & $1,91 \mathrm{~S}$ & 1,73 MR & $1,87 \mathrm{~S}$ \\
\hline CNC 0434 & $2,11 \mathrm{~S}$ & $1,72 \mathrm{MR}$ & $1,92 \mathrm{~S}$ & $1,78 \mathrm{~S}$ & $1,86 \mathrm{~S}$ & $1,75 \mathrm{~S}$ & $1,86 \mathrm{~S}$ \\
\hline TE90-180-10F & $2,12 \mathrm{~S}$ & $1,83 \mathrm{~S}$ & $1,87 \mathrm{~S}$ & $1,75 \mathrm{~S}$ & $1,87 \mathrm{~S}$ & 1,73 MR & $1,86 \mathrm{~S}$ \\
\hline IT89KD-260 & $2,12 \mathrm{~S}$ & $1,77 \mathrm{MR}$ & $1,87 \mathrm{~S}$ & 1,61 MR & $1,93 \mathrm{~S}$ & 1,67 MR & 1,83 MR \\
\hline IT89KD-245 & 1,94 MR & 1,74 MR & 1,79 MR & 1,68 MR & 1,44 MR & 1,68 MR & 1,71 MR \\
\hline
\end{tabular}

${ }^{1} \mathrm{AR}=$ altamente suscetível; $\mathrm{S}=$ suscetível; $\mathrm{MR}=$ moderadamente resistente.

${ }^{2} \mathrm{~A}$ separação foi efetuada com base no intervalo de confiança (IC).

Tabela 6 - Correlações ( $r$ ) entre os parâmetros obtidos de C. maculatus, em genótipos de caupi, por seis gerações sucessivas. Temperatura: $27,1 \pm 0,9^{\circ} \mathrm{C}$; UR: $66,6 \pm 4 \%$; Fotofase: $12 \mathrm{~h}$.

\begin{tabular}{|c|c|c|c|c|c|c|}
\hline \multirow{2}{*}{ Parâmetro } & \multicolumn{6}{|c|}{ Geração } \\
\hline & $1^{a}$ & $2^{a}$ & $3^{a}$ & $4^{a}$ & $5^{a}$ & $6^{a}$ \\
\hline & \multicolumn{6}{|c|}{ Número de ovos por fêmea } \\
\hline Viabilidade de ovos & 0,160 & $-0,387$ & 0,587 & 0,286 & $-0,042$ & $-0,165$ \\
\hline Duração da fase imatura & $-0,317$ & $-0,160$ & $-0,132$ & $-0,203$ & 0,125 & $-0,196$ \\
\hline Viabilidade da fase imatura & 0,260 & 0,143 & $-0,104$ & $-0,147$ & 0,091 & 0,009 \\
\hline \multirow[t]{2}{*}{ Índice de resistência } & 0,355 & 0,142 & $-0,039$ & $-0,105$ & 0,081 & 0,026 \\
\hline & \multicolumn{6}{|c|}{ Viabilidade de ovos } \\
\hline Duração da fase imatura & $-0,250$ & $-0,021$ & 0,367 & 0,527 & 0,064 & $-0,420$ \\
\hline Viabilidade da fase imatura & $-0,009$ & 0,309 & 0,004 & $-0,428$ & $-0,347$ & 0,595 \\
\hline \multirow[t]{2}{*}{ Índice de resistência } & 0,092 & 0,321 & $-0,066$ & $-0,459$ & $-0,351$ & 0,620 \\
\hline & \multicolumn{6}{|c|}{ Duração da fase imatura } \\
\hline Viabilidade da fase imatura & $-0,518$ & 0,309 & 0,009 & $-0,379$ & $-0,530$ & $-0,551$ \\
\hline \multirow[t]{2}{*}{ Índice de resistência } & $-0,741^{*}$ & 0,321 & $-0,294$ & $-0,472$ & $-0,595$ & $-0,638^{*}$ \\
\hline & \multicolumn{6}{|c|}{ Viabilidade da fase imatura } \\
\hline Índice de resistência & $0,954^{*}$ & $0,979^{*}$ & $0,950^{*}$ & $0,994^{*}$ & $0,997^{*}$ & $0,992^{*}$ \\
\hline
\end{tabular}

${ }^{1}$ Correlação significativa pelo teste "t" $(P \leq 0,05)$. 
a resistência destas é derivada basicamente de TVu 2027, o que resultaria em uma durabilidade da resistência relativamente baixa e o questionamento de seu valor em programas de melhoramento genético. Messina \& Renwick (1985), avaliando genótipos derivados de TVu 2027 e uma testemunha suscetível, durante seis gerações, em relação à resistência a quatro biótipos de C. maculatus, verificaram que a variação entre os mesmos foi pequena, embora o biótipo Nigeriano (mantido na criação por mais de 30 gerações) pareceu desenvolver-se mais rapidamente do que os outros nos grãos resistentes. O tempo de desenvolvimento em todas as populações, decresceu ligeiramente ou não, após as gerações de seleção. Devido todos os genótipos derivados de TVu 2027 terem retardado o desenvolvimento dos biótipos de $C$. maculatus avaliados, os autores concluíram que a frequência de ocorrência natural de populações adaptadas a estes ainda é baixa, fato também confirmado por Shade et al. (1996) após 53 gerações de seleção em laboratório.

Schoonhoven et al. (1983) e Barbosa (1997), trabalhando com o bruquídeo Zabrotes subfasciatus (Boh.) em grãos resistentes de feijão comum Phaseolus vulgaris $L$. por cinco e quatro gerações, respectivamente, não observaram perda da resistência. No presente trabalho, IT89KD-245 e IT89KD-260 mantiveram a resistência estável ao longo das gerações, não indicando adaptação do inseto. Estudos visando determinar as causas da resistência observada nestas linhagens devem ser realizados a posteriori.

\section{AGRADECIMENTOS}

À CAPES e ao CNPq pela concessão das bolsas de Mestrado e de Produtividade em Pesquisa ao primeiro e segundo autores deste trabalho, respectivamente. Ao pesquisador Dr. Francisco Rodrigues Freire Filho da EMBRAPA/CPAMN, pelo fornecimento das sementes dos genótipos de caupi. À Empresa Pernambucana de Pesquisa Agropecuária - IPA pela cessão de área da Estação Experimental Luiz Jorge da Gama Wanderley, para multiplicação das sementes.

\section{REFERÊNCIAS BIBLIOGRÁFICAS}

BAKER, T.A.; NIELSEN, S.S.; SHADE, R.E.; SINGH, B.B. Physical and chemical attributes of cowpea lines resistant and susceptible to Callosobruchus maculatus (F.) (Coleoptera: Bruchidae). Journal of Stored Products Research, v.25, p.1-8, 1989

BARBOSA, F.R. Resistência a Zabrotes subfasciatus (Boh., 1833) (Coleoptera - Bruchidae), conferida pela proteína arcelina em feijoeiro (Phaseolus vulgaris L.). Goiana, 1997. 92p. Tese (Doutorado) - Universidade Federal de Goiás.
BARROS, R.; VENDRAMIM, J.D. Efeito de cultivares de repolho, utilizadas para criação de Plutella xylostella (L.) (Lepidoptera: Plutellidae), no desenvolvimento de Trichogramma pretiosum Riley (Hymenoptera: Trichogrammatidae). Anais da Sociedade Entomológica do Brasil, v.28, p.469-476, 1999.

BECK, S.D.; SCHOONHOVEN, L.M. Conducta de los insectos y resistencia vegetal. In: MAXWELL, F.G.; JENNINGS, P.R. (Ed.) Mejoramiento de plantas resistentes a insectos. México: Editorial Limusa, 1984. p.135156.

CHAVES, J.W.N.; VENDRAMIM, J.D. Não-preferência para oviposição e desenvolvimento de Callosobruchus maculatus (Fabr.) (Coleoptera: Bruchidae) em cultivares de caupi. Anais da Sociedade Entomológica do Brasil, v.24, p.239-245, 1995.

DICK, K.M.; CREDLAND, P.F. Changes in the response of Callosobruchus maculatus (F.) (Coleoptera: Bruchidae) to a resistant variety of cowpea. Journal of Stored Products Research, v.22, p.227-233, 1986a.

DICK, K.M.; CREDLAND, P.F. Variation in the response of Callosobruchus maculatus (F.) to a resistant variety of cowpea. Journal of Stored Products Research, v.22, p.43-48, $1986 \mathrm{~b}$.

INTERNATIONAL INSTITUTE OF TROPICAL AGRICULTURE. Annual Report for 1980. Ibadan: IITA, 1981. p.117-137.

INTERNATIONAL INSTITUTE OF TROPICAL AGRICULTURE. Annual Report for 1989/90. Ibadan: IITA, 1990. p.51-59.

KORNEGAY, J.; CARDONA, C.; POSSO, C.E. Inheritance of resistance to mexican bean weevil in common bean, determined by biossay and biochemical tests. Crop Science, v.33, p.589-594, 1993.

MBATA, G.N. Evaluation of susceptibility of varieties of cowpea to Callosobruchus maculatus (F.) and Callosobruchus subinnotatus (Pic.) (Coleoptera: Bruchidae). Journal of Stored Products Research, v.29, p.207-213, 1993.

MESSINA, F.J.; RENWICK, J.A.A. Resistance to Callosobruchus maculatus (Coleoptera: Bruchidae) in selected cowpea lines. Environmental Entomology, v.14, p.868-872, 1985.

OFUYA, T.I.; CREDLAND, P.F. Responses of three populations of the seed beetle, Callosobruchus maculatus (F.) (Coleoptera: Bruchidae), to seed resistance in selected varieties of cowpea, Vigna unguiculata (L.) Walp. Journal of Stored Products Research, v.31, p.17-27, 1995.

PESSOA, G.P.; BARROS, R.; OLIVEIRA, J.V. Avaliação da resistência de caupi Vigna unguiculata (L.) Walp. a Callosobruchus maculatus (Fabr.) em confinamento em laboratório. Anais da Sociedade Entomológica do Brasil, v.22, p.259-266, 1993.

REDDEN, R.J.; MCGUIRE, J. The genetic evaluation of bruchid resistance in seed of cowpea. Australian Journal of Agricultural Research, v.34, p.707-716, 1983.

REDDEN, R.J.; DOBIE, P.; GATEHOUSE, A.M.R. The inheritance of seed resistance to Callosobruchus maculatus $F$. in cowpea (Vigna unguiculata L. Walp.): I Analysis of parental, $F_{1}, F_{2}, F_{3}$ and backcross seed generations. Australian Journal of Agricultural Research, v.34, p.681-695, 1983.

SANTOS, J.H.R. Aspectos da resistência de cultivares de Vigna sinensis (L.) Savi ao ataque do Callosobruchus maculatus (Fabr., 1775) (Col., Bruchidae), mantidos no Estado do Ceará - Brasil. Piracicaba, 1976. 194p. Tese (Doutorado) - Escola Superior de Agricultura "Luiz de Queiroz", Universidade de São Paulo.

SCHOONHOVEN, A.V.; CARDONA, C.; VALOR, J. Resistance to the bean weevil and the Mexican bean weevil (Coleoptera: Bruchidae) in noncultivated commom bean accessions. Journal of Economic Entomology, v.76, p.1255-1259, 1983.

SCOTT, A.J.; KNOTT, M.A. A cluster analysis method for grouping means in the analysis of variance. Biometrics, v.30, p.507-512, 1974.

SECK, D. Resistance to Callosobruchus maculatus F. (Col., Bruchidae) in some cowpea varieties from Senegal. Journal of Stored Products Research, v.29, p.49-52, 1993.

SHADE, R.E.; KITCH, L.W.; MENTZER, P.; MURDOCK, L.L. Selection of a cowpea weevil (Coleoptera: Bruchidae) biotype virulent to cowpea weevil resistant Landrace TVu 2027. Journal of Economic Entomology, v.89, p.1325-1331, 1996.

SINGH, S.R. Resistance to pest of cowpea in Nigeria. In: SINGH, S.R.; VAN EMDEN, H.F.; TAYLOR, T.A. (Ed.) Pests of grain legumes: ecology and control. London: Academic Press, 1978. p.267-279.

SINGH, B.B.; SINGH, S.R.; ADJADI, O. Bruchid resistance in cowpea. Crop Science, v.25, p.736-739, 1985.

$\overline{\text { Recebido em } 28.11 .00}$ 